\title{
Pediatric Extracorporeal Shockwave Lithotripsy: Its Efficiency at Various Locations in the Upper Tract
}

\author{
Fayez T. Hammad, FRCS., Ph.D., FRACS, ${ }^{1}$ Mohammed Kaya, M.D., ${ }^{2}$ and Essa Kazim, M.D., FRCS ${ }^{2}$
}

\begin{abstract}
Background: Several reports demonstrated the effectiveness of extracorporeal shockwave lithotripsy (SWL) in the management of pediatric upper tract urolithiasis. The majority of these studies used the stone-free rate as the main indicator for treatment success. Efficacy Quotient (EQ) is an important outcome measure because it not only takes into consideration the stone-free rate, but also includes other undesirable outcomes that increase patient morbidity and utilizes hospital resources. Nevertheless, very few studies reported on the EQ of pediatric SWL at various locations in the upper tract.

Methods: We retrospectively reviewed the records of 84 children (103 urinary calculi) who were treated with SWL in our institution between 1995 and 2005.

Results: There were 84 renal (pelvis, $n=27$; upper calyx, $n=13$; middle calyx, $n=8$; lower calyx, $n=36$ ) and 19 ureteric (upper third, $n=10$; middle third, $n=5$; lower third $n=4$ ) stones. EQ for renal pelvic stones was 67\% compared to $48 \%$ for caliceal stones (upper calyx: 52\%, middle calyx: $46 \%$ and lower calyx: $47 \%$ ). Overall, the EQ for renal stone was $54 \%$ and it was $59 \%$ for ureteric stones. This efficiency in the ureter was achieved at the expense of a higher number of shock waves delivered (3606 vs. 2592, $P<0.01$ ). Overall, the stone-free rate was $82 \%$ (renal, $81 \%$; ureteric calculi $84 \%$ ). The only factor that affected the stone-free rate was the size of the stone (33\% in stones $>2 \mathrm{~cm}$ and $87 \%$ in stones $<2 \mathrm{~cm}(P<0.01)$. The overall retreatment rate was $41 \%$ (renal, $40 \%$ and ureteric $42 \%$ ). However, the number of total treatments ranged from 1 to 5 sessions in renal stones and 1 to 2 sessions in ureteric stones. Auxiliary procedures were performed in 9 patients (stenting, $n=3$; ureteroscopy, $n=4$ and percutaneous nephrolithotomy, $n=2$ ).

Conclusions: Pediatric SWL appears to be more efficient in the renal pelvic stones compared to caliceal stones. The efficiency of SWL in the ureter is achieved with a higher number of shock waves delivered. A discussion on the possible future modifications of the definition of EQ, especially in children, is also provided.
\end{abstract}

\section{Introduction}

$\mathbf{P}$ EDIATRIC UROLITHIASIS is a rare entity that is being recognized more frequently. ${ }^{1,2}$ The management of upper tract stones in children is still challenging and continues to evolve due to the continuous advances in technology. Due to its high acceptance among patients and physicians, extracorporeal shockwave lithotripsy (SWL) has emerged as one of the first-line minimally invasive treatment techniques for pediatric upper tract urolithiasis despite the several technical limitations and possible harmful effects on the growing organs of children.
Several reports have demonstrated the effectiveness of SWL in the management of pediatric urolithiasis. ${ }^{3-11}$ The majority of these studies used the stone-free rate as the main indicator for treatment success. ${ }^{3-7}$ Despite the fact that stonefree status is the ultimate goal of any stone treatment modality, it is important also to measure the Efficacy Quotient (EQ), because it also takes into consideration factors that increase patient's morbidity and anxiousness and result in waste of patient time and hospital resources such as the retreatment rate and the rate of auxiliary procedures. Despite this, only very few studies have reported on the EQ as an important indicator of treatment success in the upper tract

\footnotetext{
${ }^{1}$ Departnent of Surgery, Faculty of Medicine, Al Ain, United Arab Emirates.

${ }^{2}$ Department of Urology, Dubai Hospital, Dubai, United Arab Emirates.
} 
urolithiasis..$^{8-10,12}$ The majority of these studies reported on the EQ either in renal ${ }^{9,10}$ or ureteric stones. ${ }^{12}$ One of the very few studies that reported on the EQ in both locations was the one by Muslumanoglu and colleagues. ${ }^{8}$ Nevertheless, their study reported on the EQ in different parts on the ureter, renal pelvis, and calyces, and there was no report on the EQ in stones at various caliceal locations. In this study, we retrospectively analyzed the efficacy of SWL for upper tract stones in various locations in the pediatric age group at our institution. In addition, a discussion on the possible future modifications of the definition of EQ, especially in children, is also provided.

\section{Patients and Methods}

A total of 2913 patients with urinary tract stones underwent SWL at our institute between January 1995 and December 2005 using the Dornier MFL-5000 Lithotripter. Out of those, 93 patients (3.2\%) were younger than 18 years and were considered for the study. The medical records of 90 patients were available for this retrospective review. Patients with congenital abnormalities $(n=3)$ or neurogenic bladder $(n=1)$ were excluded. In addition, 2 patients were also excluded because they had radiolucent stones and thus their preoperative diagnosis, intraoperative identification, and postoperative follow-up with ultrasound only is different from the majority of the group. The remaining 84 patients were included in the study. There were $38(45 \%)$ boys and $46(55 \%)$ girls with a mean age of 10.1 years (range: $4-17$ years).

The data collected included patients' clinical presentation; past history of stone disease or stone surgery; family history of stone disease; preoperative blood, urine, and radiological investigations; stone(s) number, size, and location including the presence of underlying urinary tract abnormalities; and data related to the SWL procedures and their outcomes.

The stones were stratified according to their location and size of the maximum diameter into less than $1 \mathrm{~cm}, 1-2 \mathrm{~cm}$, and larger than $2 \mathrm{~cm}$. Patients' follow-up included plain radiography, 1 day, and 1-3 months postoperatively. Followup ultrasound was performed when indicated, especially in the presence of post-SWL complications such as renal colic resistant to oral medications. SWL procedures with no evidence of fragmentation were considered unsuccessful. Patients with fragments $4 \mathrm{~mm}$ or greater were usually consid- ered for further SWL. Asymptomatic, non-infectious, and nonobstructive fragments smaller than $4 \mathrm{~mm}$ were considered clinically insignificant residual fragments (CIRFs). Complete stone removal (stone-free) was defined as the lack of any visible stone fragments on radiological studies up to 3 months following SWL treatment. When a single SWL session was performed for a group of small stones lying adjacently (e.g., in a calyx), the size of the stone was calculated by adding the diameter of these stones.

The proportions of various parameters were calculated according to their relevant group. For example, stone-free and retreatment rates were calculated on stone basis, whereas the number of shock waves delivered was calculated on a session basis. Similarly, clinical presentation and complications were calculated on a patients' basis.

The Efficiency Quotient (EQ) was calculated using the following equation as previously described ${ }^{13}$ :

$\mathrm{EQ}=(\%$ stone free $/(100 \%+\%$ retreatment

$$
+\% \text { auxiliary procedures) })^{*} 100
$$

The collected data were entered and analyzed using Microsoft Excel and SPSS. $\chi^{2}$ and Fisher's exact tests were used to compare retreatment and stone-free rates and the incidence of CIRFs as appropriate. The $z$ test was used to compare the mean number of shockwaves delivered to renal and ureteric stones. The multivariate logistic regression test was used to determine the effect of stone size and location on the stone-free rate. A $P$ value of $<0.05$ was considered statistically significant.

\section{Results}

A total of 103 calculi in $87 \mathrm{renal} /$ ureteric tracts (84 patients) were treated requiring 156 SWL sessions. Forty-eight calculi $(47 \%)$ were in the right side compared to 55 calculi (53\%) found in the left side.

Table 1 summarizes stone size and distribution data. There were 84 renal and 19 ureteric stones. The renal stones were located in the pelvis in 27 cases and in a calyx in 57 cases. More than half of the renal stones (52\%) were less than $1 \mathrm{~cm}$ in diameter and only $11 \%$ of them were larger than $2 \mathrm{~cm}$ in diameter.

Of the ureteric stones, 10 were in the proximal third, 5 in the mid third, and 4 in the lower third of the ureter. Only 2 ureteric stones were larger than $1 \mathrm{~cm}$ in diameter with a max-

Table 1. Stone Size and Distribution Data

\begin{tabular}{lrcccc}
\hline Stone location & No. & $\begin{array}{c}\text { No. stones } \\
<1 \mathrm{~cm}\end{array}$ & $\begin{array}{c}\text { No. stones } \\
1-2 \mathrm{~cm}\end{array}$ & $\begin{array}{c}\text { No. stone } \\
>2 \mathrm{~cm}\end{array}$ & $\begin{array}{c}\text { Mean size } \\
\text { (range mm) }\end{array}$ \\
\hline Renal & 86 & 45 & 32 & 9 & $10.3(5-24)$ \\
$\quad$ Pelvis & 28 & 19 & 4 & 5 & 9 \\
Upper calix & 13 & 7 & 6 & 0 & 11 \\
Middle calix & 8 & 3 & 4 & 1 & 9 \\
Lower calix & 37 & 16 & 18 & 3 & 11 \\
Ureter & 19 & 17 & 2 & 0 & $9.8(7-13)$ \\
Upper Ureter & 10 & 8 & 0 & 0 & 9 \\
Middle Ureter & 5 & 5 & 0 & 0 & 8 \\
Lower Ureter & 4 & 4 & 34 & 9 & $10.0(5-24)$ \\
Total & 105 & 62 & & & 9 \\
\hline
\end{tabular}




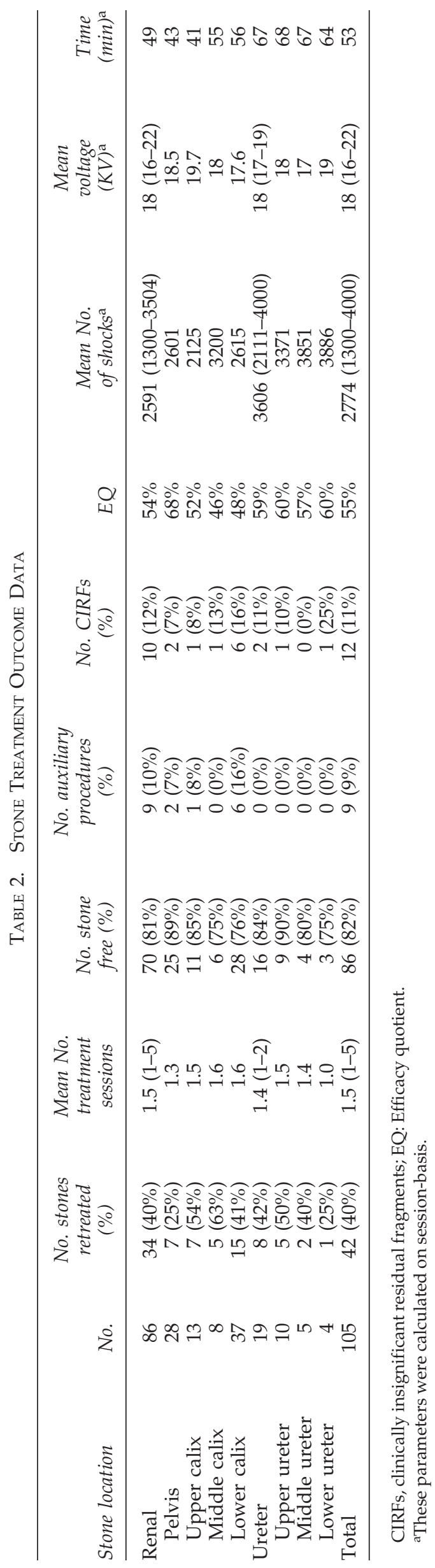


imum size of $1.3 \mathrm{~cm}$. Both stones were in the proximal ureter. None of the children who were treated with SWL had bladder calculi.

The EQ of SWL at various locations of the upper tract is shown in Table 2. Within the kidney, the EQ for pelvic stones was $67 \%$, whereas it was $48 \%$ for caliceal stones (upper calyx: $52 \%$, middle calyx: $46 \%$, and lower calyx: $47 \%$ ). Overall, the EQ for renal stones was $54 \%$ and it was $59 \%$ for ureteric stones. This efficiency in the ureter was achieved at the expense of a higher number of shock waves delivered (3606 vs. $2592, P<0.01)$. Overall, the number of sessions according to the number of shockwaves (SWs) delivered was as follows: 1300-2000 SWs, $\mathrm{n}=59 ;$ 2000-3000 SWs, $\mathrm{n}=52 ; 3000-4000$ SWs, $n=45$. In our institution, the maximum number of SWs that can be delivered at a single session should not exceed $4000 \mathrm{SWs}$ at a rate of $120 \mathrm{SWs} /$ minute.

Overall, the stone-free rate was $82 \%$. The stone-free rates in both renal and ureteric calculi were comparable $(81 \%$ and $84 \%$, respectively, $P=0.74)$. Using a univariate analysis, the only factor that affected the stone-free rate was the size of the stone (the stone-free rate in stones larger than $2 \mathrm{~cm}$ was $33 \%(3 / 9)$ compared to $87 \%(82 / 94)$ in those smaller than 2 $\mathrm{cm}(P<0.01)$. Similar results were obtained using the multivariate logistic regression (stones $<2 \mathrm{~cm}$ : B (regression coefficient) 1.09 , odds ratio 2.1, 95\% confidence interval (CI) 1.32-7.55, $P<0.05)$. The stone location was not a predictor of the stone-free rate (pelvic vs. caliceal: B 0.72, odds ratio 1.30, 95\% CI 1.07-13.48, $P=0.12$; ureteric vs. renal: B 0.97, odds ratio $1.13,95 \% \mathrm{CI} 1.14-11.02, P=0.09$ ).

Further SWL treatment was needed in $41 \%$ of the stones ( 1 session, $\mathrm{n}=61 ; 2$ sessions, $\mathrm{n}=34 ; 3$ sessions, $\mathrm{n}=6$; 4 sessions, $\mathrm{n}=1$; 5 sessions, $\mathrm{n}=1$ ) with no difference in the retreatment rate between renal and ureteric stones $(40 \%$ and $42 \%$, respectively, $P=0.84$ ). However, the number of total number of treatment sessions varied from 1 to 5 sessions in the renal stones compared to a maximum of 2 sessions in ureteric stones. Within the kidney, the proportion of stones that needed retreatment was higher in caliceal stones when compared to pelvic stones ( $47 \%$ vs. $26 \%, P<0.05$ ).

There was no difference in the incidence of CIRFs in both renal and ureteric stones $(12 \%$ and $11 \%$, respectively, $P=0.41)$ or between pelvic and caliceal stones $(7 \%$ and $14 \%$, respectively, $P=0.09$ ).

Pre-SWL stenting was required in 9 cases with the stones larger than $2 \mathrm{~cm}$. Following SWL, auxiliary procedures were needed in 9 patients (stenting, $\mathrm{n}=3$; ureteroscopy, $\mathrm{n}=4$; and percutaneous nephrolithotomy, $\mathrm{n}=2$ ). Complications were observed in 11 patients (13\%) in the form of renal colic $(n=8)$ and urinary tract infections $(n=3)$. Three of the patients with renal colic required stenting and 4 required ureteroscopy. No significant dermal ecchymosis was reported in any patient. Self-limiting hematuria was reported in 70 patients.

In our institute, general anaesthesia is used for children 12 years and older unless the family chooses otherwise. Thirty-five sessions (24\%) were performed with patients under general anaesthesia and 121 (76\%) with patients under sedation, mainly in the form of midazolam and propofol. General anaesthesia was used more commonly in younger patients and sedation was used mainly in older children (mean age: 8.1 years [range: $4-13$ ] vs. 12.8 years [range: 5-17], respectively).
The presenting symptoms included renal colic/abdominal pain $(n=78)$, lower urinary tract symptoms $(n=14)$, and urinary tract infections $(n=10) ; 4$ of them were in the form of pyelonephritis, hematuria $(\mathrm{n}=11)$, and scrotal pain $(\mathrm{n}=$ 2 ). Twenty-seven patients (32\%) had 2 or more presenting symptoms, whereas the remaining 57 patients $(68 \%)$ presented with only one of the clinical pictures mentioned above. Radiological investigations performed were intravenous urogram in 64 patients, ultrasonography in 15 patients, and both in 7 patients. All patients had pre-SWL plain KUB x-ray, and none of them had a noncontrast CT scan.

All except 2 patients had normal serum creatinine. Eighteen patients had positive urine cultures requiring treatment prior to SWL and perioperative prophylaxis as per urine culture sensitivity results.

History of previous spontaneous stone passage was found in 14 patients. In addition, 13 patients gave history of stone surgery prior to SWL including ureteroscopy, pyelolithotomy, and percutaneous pyelolithotomy. Eleven patients had SWL for other stones prior to the study period. Family history of stone disease was reported in 22 patients.

The mean follow-up was 11.3 months (range: 1-62 months). During the follow-up time, the blood pressure was normal in all patients as was the case prior to the SWL.

\section{Discussion}

Pediatric urolithiasis is a rare entity that was shown to have an increasing frequency. ${ }^{1,2}$ The treatment of this condition in general, and of the upper tract stones in particular, is still challenging despite the recent and ongoing advances in medical technology, especially in the field of fiberoptics. This is probably due to several factors, the most important of which is the small size of the urinary organs in children. Among all the treatment options, SWL has a relatively high acceptance among both patients and physicians. This is probably due to the fact that SWL can be repeated relatively easily in the same patient and the fact that the same machine can be used in both adults and children, making it an attractive option in centers with limited resources.

The majority of studies that demonstrated the effectiveness of SWL in the management of pediatric upper tract urolithiasis used the stone-free rate as the main indicator for treatment success, ${ }^{3-7}$ with only a limited number of studies reporting on the EQ at various locations. ${ }^{8-10,12}$ The importance of the EQ can be demonstrated by the fact that it is theoretically possible to achieve a $100 \%$ stone-free rate in the majority of upper tract stones in children by exposing the patient to repeated treatment sessions and auxiliary procedures. However, this is inappropriate not only due to the increase in patient morbidity and waste of hospital resources, but also due to the possible harmful effects of the SWL on the growing organs of the children. ${ }^{14,15}$ Therefore, the calculation of EQ in this age group in particular is probably more important than in adults, especially when counseling parents regarding the treatment consequences. In the current study, we calculated the EQ at various parts in the upper tract including upper, middle, and lower calices.

The efficiency or efficacy of any treatment modality is measured by its ability to produce the desired result with the least waste. In SWL, the term Efficacy Quotient (EQ) was 
introduced as an indicator of its efficiency and was used to compare the results of SWL with other stone treatment modalities for various stone locations and among several institutions. ${ }^{13}$ The equation that defines the EQ has included the desired outcome (i.e., the stone-free rate in the numerator) and the degree of "waste" (i.e., the percentage of retreatment rate and the auxiliary procedures in the denominator). In the current study, the efficiency of SWL appears to be better for renal pelvic compared to caliceal stones (EQ: $67 \%$ vs. $48 \%$, respectively). The reason for this trend is difficult to explain from the results of this study; however, it is well known that the transmission of ultrasonic waves is better in the fluid media compared to solid media. Therefore, it is possible that their efficiency in the renal pelvis where the stone is usually surrounded by urine is better than in the calyx where the amount of surrounding urine is less. Another possible factor is the relative difficulty of drainage of residual stones from calices, especially lower pole calices, compared to other locations due to the inherent variation in the architecture of the pelvicaliceal system and gravity factors. ${ }^{16,17}$

In the current study, the EQ of SWL for ureteric stones is $59 \%$ compared to $54 \%$ for renal stones. Similar trends were reported by the Muslumanoglu et al. group. ${ }^{8}$ The efficiency of SWL in the treatment of ureteric stones depends on several factors. For instance, the shorter distance traveled by the stone could only be one of the factors that explain the relative ease of passage of ureteric residual fragments compared to renal pelvic fragments. On the other hand, the fact that ureteric stones are not surrounded by urine would probably decrease the efficiency of SWL in the ureter. The mobility of the ureter due to peristalsis may also have a similar effect. At any rate, the relatively high efficiency of the SWL in the ureter appears to be achieved at the expense of delivering a higher number of shockwaves as in this study. Therefore, factors such as the number of shockwaves and the voltage should also be included when calculating the EQ because the higher the number of shockwaves and voltage, the higher the energy and time consumed, as demonstrated by the extra time used to treat ureteric compared to renal stones in this study (67 vs. 49 minutes, $P<0.01$ ). This is particularly important when describing the EQ in children because there is evidence, at least from animal studies, to suggest dose-related renal damage following SWL, ${ }^{14,15}$ especially in younger individuals. ${ }^{18}$ Obviously, further clinical studies are needed to determine the optimal number of shockwaves and voltage that result in least damage. With this regard, da Cunha Lima and associates have demonstrated that a voltage of 14 $\mathrm{kV}$ is as effective in stone fragmentation as higher voltages; ${ }^{7}$ therefore, any voltage that is higher than $14 \mathrm{kV}$ may be considered to decrease the overall efficiency of that particular treatment. Further studies are required to confirm these findings. Alternatively, the use of the total energy delivered in energy units will probably be more useful than using individual factors such as the total number of shockwaves and voltage, especially when comparing different machines; however, this may not be easy, especially among machines in which the total amount of energy is not provided, and further prospective research is needed in this field.

Other factors that affect the efficiency, such as the percentage of the CIRFs, should also probably be included when calculating EQ because there is a growing body of evidence to suggest that CIRFs are not really insignificant, and up to
$34 \%$ of them may grow to significant stones with time. ${ }^{19,20}$ Even with the relatively short period of mean follow-up in the current study, 2 of CIRFs have increased in size and required further intervention. The rest either passed spontaneously or did not increase in size. With this modification, the equation would be as follows:

$$
\begin{aligned}
& \mathrm{EQ}=(\% \text { stone free } /(100 \%+\% \text { retreatment } \\
& \qquad \% \% \text { auxiliary procedures }+\% \text { CIRFs }))^{*} 100
\end{aligned}
$$

In the current study, this modification would not result in major changes because the modified EQ for pelvic, upper caliceal, middle caliceal, lower caliceal, upper ureteric, middle ureteric, and lower ureteric stone would be $63 \%, 50 \%$, $43 \%, 43 \%, 56 \%, 57 \%$, and $50 \%$, respectively. This is probably due to the relatively small numbers of stones in each group. However, in studies with large number of patients such as the one reported by Muslumanoglu et al, 8 the inclusion of CIRFs would increase the difference between the EQ of pelvic and caliceal stones, an issue that is important in planning treatment and probably in counseling patients with stones at different locations. Therefore, despite the fact that inclusion of the CIRF in the EQ definition may pose some problems related to the definition of the cutoff size and the methodology of measuring the residual fragments, the incorporation of CIRF appears to be important for a more accurate definition of the parameter.

In this study, an overall stone-free rate of $82 \%$ was achieved. The stone-free rate was not affected by stone location because there was no difference between ureteric and renal stones nor was there a difference between stone-free rate in different sites of the kidney or ureter. The only factor that affected the stone-free status was the size of the stone. These results were in agreement with the previously published data. 8,11 This probably explains, at least partially, the lack of differences in stone-free rate according to stone location because there was no difference in the stone size between different locations (e.g., mean size of renal stones was $10.3 \mathrm{~mm}$ vs. $9.8 \mathrm{~mm}$ for ureteric stones).

In the current study, the retreatment rate and the mean number of treatment sessions were similar in both renal and ureteric stones. This is also probably due to the fact that there was no difference in the size of the renal and ureteric stones $(P=0.19)$. However, the range of retreatment sessions in the renal stones was 1-5 sessions, whereas ureteric stones required a maximum of 2 sessions. This discrepancy is most probably due to the greater variation in the size of renal stones compared to the ureteric ones $(5-24 \mathrm{~mm}$ for renal stones vs. 7-13 $\mathrm{mm}$ in ureteric stones). Other possible reason for the ureteric stones to have a maximum of 2 sessions is the availability of a good pediatric ureteroscopic facility at our institution, which probably resulted in patients choosing to undergo ureteroscopic stone fragmentation after failure of 1or 2 SWL sessions.

The proportion of stones that needed retreatment was higher in caliceal stones when compared to pelvic stones. This may imply that similar stone-free rates between the two locations were achieved at the expense of a higher retreatment rate in the former. This finding is in agreement with the findings of some reports. ${ }^{3}$ Other studies, on the contrary, reported almost similar retreatment rates between pelvic and caliceal stones. ${ }^{8-10}$ It is difficult to explain this discrepancy between different studies, but a possible explanation, at least 
in our study, would be the possible awareness of the relative difficulty in managing the residual caliceal stones compared to pelvic stones, especially the endoscopic treatments including percutaneous nephrolithotomy in this age group. In addition, it is well known that the drainage of residual stones from the calices, especially the lower calices, is probably more difficult than in other locations. ${ }^{16,17}$ This might have resulted in a more aggressive approach in dealing with caliceal stones in a trial to render them stone free. Collectively, this indicates that, at least in some studies like our study, the comparable stone-free rates between different locations such as pelvis and calices were achieved at the expense of higher retreatment rates in the latter, resulting in a lower EQ of SWL in this location (EQ for pelvic stones is $67 \%$ vs. $48 \%$ for caliceal stones).

The current study, which has the inherent problems of retrospective studies, did not particularly address the immediate or long-term possible renal damage caused by the SWL treatment. However, there appear to be no major immediate postoperative complications, which occurred only in $13 \%$ of patients in the form of self-limited renal colic and urinary tract infections. In addition, with the relatively short followup, none of the patient had deterioration in blood pressure during the period of follow-up. However, from our study, we cannot rule the potential long-term complications such as hypertension and diabetes. For better evaluation of the predictors of SWL efficiency, further prospective studies with a large number of patients and uniform assessment of sones pre- and post-SWL (e.g., by using noncontrast computed tomography scan) are needed. Another caveat of the current study is the use of shockwaves at a rate of $120 / \mathrm{min}$. Obviously, this rate is slightly fast for children and currently, we use lower rates, which proved to have a higher efficiency. ${ }^{21-24}$ However, this change in policy was due to the relatively recent evidence from in vivo studies, which were mainly published after the year 2000. At the time of the study period, we and several other institutions used SWL at a rate of $120 / \min ^{23}$

In conclusion, SWL in children has satisfactory stone-free results with minimal morbidity. Pediatric SWL appears to be more efficient in the renal pelvic stones compared to caliceal stones. The efficiency of SWL in the ureter is achieved with a higher number of shock waves delivered. The treatment efficiency measured by EQ should include other potential factors such as the number of shockwaves delivered, voltage, and the rate of CIRF.

\section{Acknowledgments}

The authors would like to acknowledge Mr. S. J. Mohamed for his clinical input.

\section{Disclosure Statement}

The authors state that no competing financial interests exist.

\section{References}

1. Kroovand RL. Pediatric urolithiasis. Urol Clin North Am 1997;24:173-184.

2. VanDervoort K, et al. Urolithiasis in pediatric patients: A single center study of incidence, clinical presentation and outcome. J Urol 2007;177:2300-2305.
3. Rodrigues Netto N Jr, et al. Extracorporeal shock wave lithotripsy in children. J Urol 2002;167:2164-2166.

4. Ather $\mathrm{MH}$, Noor MA. Does size and site matter for renal stones up to $30-\mathrm{mm}$ in size in children treated by extracorporeal lithotripsy? Urology 2003;61:212-215; discussion 215.

5. Shokeir AA, et al. Treatment of renal stones in children: A comparison between percutaneous nephrolithotomy and shock wave lithotripsy. J Urol 2006;176:706-710.

6. Ozgur Tan M, et al. Extracorporeal shock-wave lithotripsy for treatment of ureteral calculi in paediatric patients. Pediatr Surg Int 2003;19:471-474.

7. da Cunha Lima JP, et al. Extracorporeal shock wave lithotripsy in children: Results and short-term complications. Int J Urol 2007;14:684-688.

8. Muslumanoglu AY, et al. Extracorporeal shock wave lithotripsy as first line treatment alternative for urinary tract stones in children: A large scale retrospective analysis. J Urol 2003;170(6 Pt 1):2405-2408.

9. Demirkesen O, et al. Efficacy of extracorporeal shock wave lithotripsy for isolated lower caliceal stones in children compared with stones in other renal locations. Urology 2006;67: 170-174; discussion 174-175.

10. Nomikos MS, Sowter SJ, Tolley DA. Outcomes using a fourth-generation lithotripter: A new benchmark for comparison? BJU Int 2007;100:1356-1360.

11. Aksoy $Y$, et al. Extracorporeal shock wave lithotripsy in children: Experience using a mpl-9000 lithotriptor. World J Urol 2004;22:115-119.

12. Muslumanoglu AY, et al. Efficacy of extracorporeal shock wave lithotripsy for ureteric stones in children. Int Urol Nephrol 2006;38:225-229.

13. Denstedt J, Clayman RV, Preminger GM. Efficiency Quotient as a means of comparing lithotriptors. J Endourol 1990;4:S100.

14. Willis LR, et al. Shockwave lithotripsy: Dose-related effects on renal structure, hemodynamics, and tubular function. J Endourol 2005;19:90-101.

15. Delius M, et al. Biological effects of shock waves: Kidney damage by shock waves in dogs-dose dependence. Ultrasound Med Biol 1988;14:117-122.

16. Elbahnasy AM, et al. Lower-pole caliceal stone clearance after shockwave lithotripsy, percutaneous nephrolithotomy, and flexible ureteroscopy: Impact of radiographic spatial anatomy. J Endourol 1998;12:113-119.

17. Lingeman JE, et al. Management of lower pole nephrolithiasis: A critical analysis. J Urol 1994;151:663-667.

18. Willis LR, et al. Relationship between kidney size, renal injury, and renal impairment induced by shock wave lithotripsy. J Am Soc Nephrol 1999;10:1753-1762.

19. El-Nahas AR, et al. Predictors of clinical significance of residual fragments after extracorporeal shockwave lithotripsy for renal stones. J Endourol 2006;20:870-874.

20. Osman MM, et al. 5-year-follow-up of patients with clinically insignificant residual fragments after extracorporeal shockwave lithotripsy. Eur Urol 2005;47:860-864.

21. Yilmaz E, et al. Optimal frequency in extracorporeal shock wave lithotripsy: Prospective randomized study. Urology 2005;66:1160-1164.

22. Weir MJ, Tariq N, Honey RJ. Shockwave frequency affects fragmentation in a kidney stone model. J Endourol 2000;14: 547-550.

23. Madbouly K, et al. Slow versus fast shock wave lithotripsy rate for urolithiasis: A prospective randomized study. J Urol 2005;173:127-130.

24. Greenstein A, Matzkin H. Does the rate of extracorporeal shock wave delivery affect stone fragmentation? Urology 1999;54:430-432. 
Address reprint requests to: Fayez T. Hammad, F.R.C.S.Ed., Ph.D., F.R.A.C.S. Department of Surgery Faculty of Medicine P.O. Box 17666 Al Ain

United Arab Emirates

E-mail: fayez@mail2doctor.com fayezh@uaeu.ac.ae

\section{Abbreviations Used}

$\mathrm{CIRF}=$ clinically insignificant residual fragments

$\mathrm{CT}=$ computed tomography

$\mathrm{EQ}=$ efficacy quotient

SWL $=$ extracorporeal shockwave lithotripsy 
